\title{
Testing and calibration of phase plates for JWST optical simulator
}

\author{
Qian Gong ${ }^{\mathrm{a}}$, Jenny Chu ${ }^{\mathrm{b}}$, Severine Tournois ${ }^{\mathrm{c}}$, William Eichhorn ${ }^{\mathrm{a}}$, David Kubalak ${ }^{\mathrm{a}}$ \\ ${ }^{\mathrm{a} N A S A}$ Goddard Space Flight Center, Greenbelt, Maryland, USA 20771; \\ borbital Sciences Corporation TSD, 7500 Greenway Center Drive, Greenbelt MD 20770; \\ 'Stinger Ghaffarian Technologies, 7701 Greenbelt Road, Suite 400, Greenbelt, Maryland 20770;
}

\begin{abstract}
Three phase plates were designed to simulate the JWST segmented primary mirror wavefront at three on-orbit alignment stages: coarse phasing, intermediate phasing, and fine phasing. The purpose is to verify JWST's on-orbit wavefront sensing capability. Amongst the three stages, coarse alignment is defined to have piston error between adjacent segments being $30 \mu \mathrm{m}$ to $300 \mu \mathrm{m}$, intermediate being $0.4 \mu \mathrm{m}$ to $10 \mu \mathrm{m}$, and fine is below $0.4 \mu \mathrm{m}$. The phase plates were made of fused silica, and were assembled in JWST Optical Simulator (OSIM). The piston difference was realized by the thickness difference of two adjacent segments. The two important parameters to phase plates are piston and wavefront errors. Dispersed Fringe Sensor (DFS) method was used for initial coarse piston evaluation, which is the emphasis of this paper. Point Diffraction Interferometer (PDI) is used for fine piston and wavefront error. In order to remove piston's 2 pi uncertainty with PDI, three laser wavelengths, $640 \mathrm{~nm}, 660 \mathrm{~nm}$, and $780 \mathrm{~nm}$, are used for the measurement. The DHS test setup, analysis algorithm and results are presented. The phase plate design concept and its application (i.e. verifying the JWST on-orbit alignment algorithm) are described. The layout of JWST OSIM and the function of phase plates in OSIM are also addressed briefly.
\end{abstract}

\section{INTRODUCTION}

OSIM is a JWST optical simulator design to simulate optical performance of the JWST and for verifying the JWST Integrated Science Instrument Module (ISIM) ${ }^{1}$. The phase plates were designed to test one of JWST's instruments: NIRCam, where key algorithms for on-orbit optical telescope segments alignment mechanisms are implemented. The goal is to make the telescope diffraction limited at $\lambda=2.12 \mu \mathrm{m}$. This not only requires diffraction limited surface figures for each segment, but also requires sub-micron piston error between segments. As we all know, how well the segments of the telescope primary mirror can be aligned determines how well the science goal of JWST can be achieved. Phase plates will be used as a reference to verify the on-orbit alignment capability. This implies the importance of the test and characterization of the phase plates.

Three phase plates were designed to test two mechanisms built into NIRCam instrument: Dispersed Hartmann Sensor (DHS) and Phase Retrieval (PR). The difference between DHS and DFS is that DHS tests the piston error of all segments simultaneously, and DFS tests one pair at a time. DHS is used to align coarser piston error just after mechanical alignment is done. PR is used to adjust fine piston error and wavefront error amongst segments. OSIM coarse phase plate was designed to verify DHS mechanism, fine phase plate to verify PR mechanism, and intermediate to see if there is a smooth transaction between the two mechanisms.

The whole phase plate test and characterization procedure includes an ambient DFS test, and an ambient and cryogenic PDI test. The reason for not doing a cryo DFS test is that the analysis shows the temperature induced piston change is not going to exceed the PDI's piston measurement range. The following DFS test was performed in optics laboratory. It will not be repeated during OSIM test, because OSIM does not have a built in DFS capability.

a Qian.gong-1@nasa.gov; phone: 1-301-286-1490; fax: 1-301-286-0402 
The DFS result of the coarse phase plate shows that the piston error less than $2 \mu \mathrm{m}$ has been achieved, which provides an excellent starting point for PDI test and characterization that was estimated to have maximum of $4 \mu \mathrm{m}$ measurement range with selected 3 wavelengths: $640 \mathrm{~nm}, 660 \mathrm{~nm}$, and $780 \mathrm{~nm}$. The result and discussion is presented in the following sections. The PDI ambient and cryo test will be performed during OSIM thermal-vacuum test in the near future. After the data is collected and analyzed, the result will be published in another paper.

\section{PHASE PLATE DESIGN AND ITS FUNCTION IN OSIM}

\subsection{Phase Plate Design}

Phase plates were designed to match the size of the JWST telescope pupil, which is also the size of the OSIM pupil. All three phase plates were made of fused silica. The phase differences (also called piston differences) were obtained by varying the thickness of each segment. For coarse phase plate, 19 hexagon segments were optically polished to different thicknesses and mechanically assembled together as shown in Figure 1 (a). The phase difference range among the pairs of the segments for coarse phase plate is $30 \mu \mathrm{m}-300 \mu \mathrm{m}$ with a uniform distribution. The phase distribution is shown in Figure 1 (b).

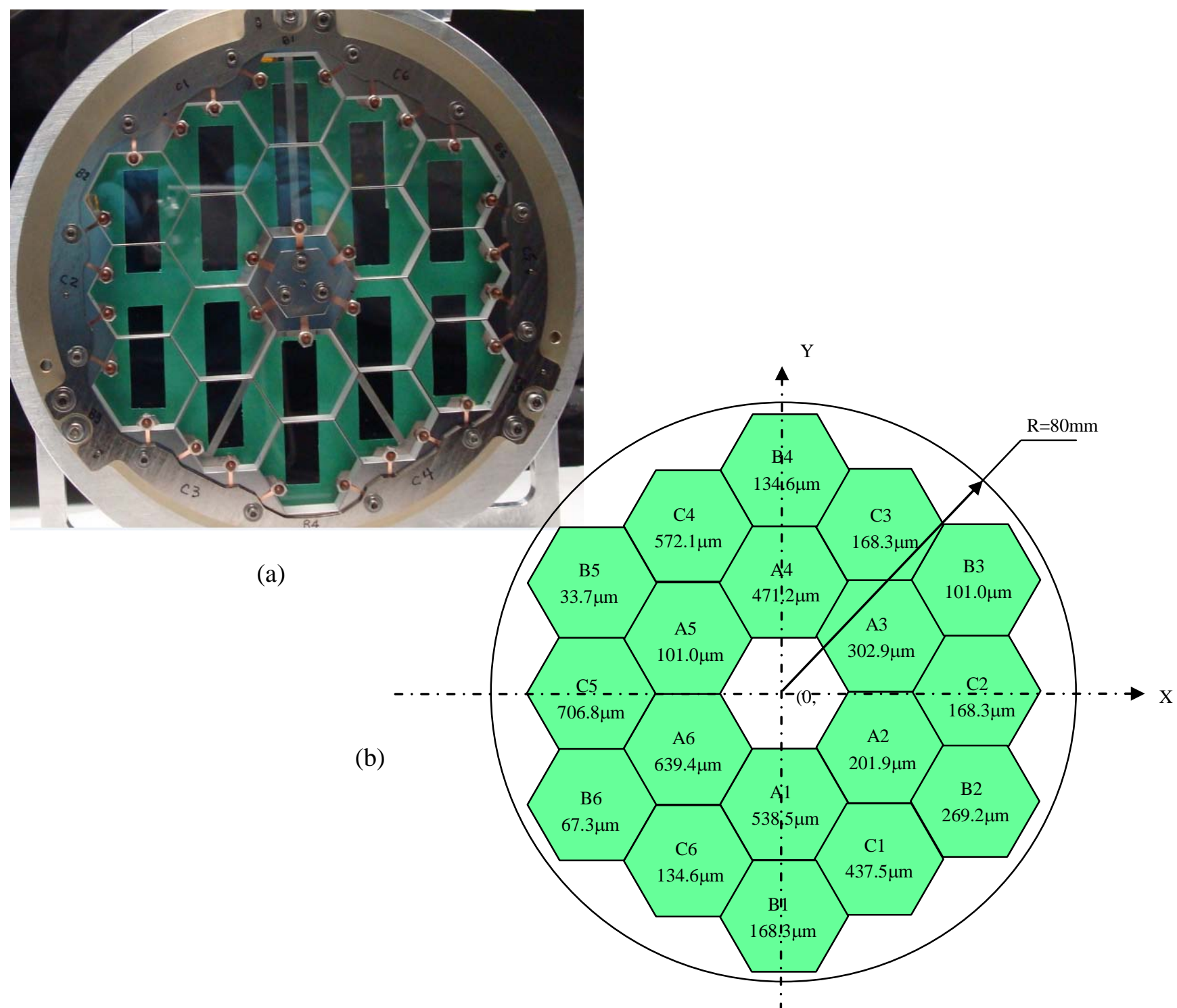

Figure 1. Coarse phase plate and its piston distribution. 


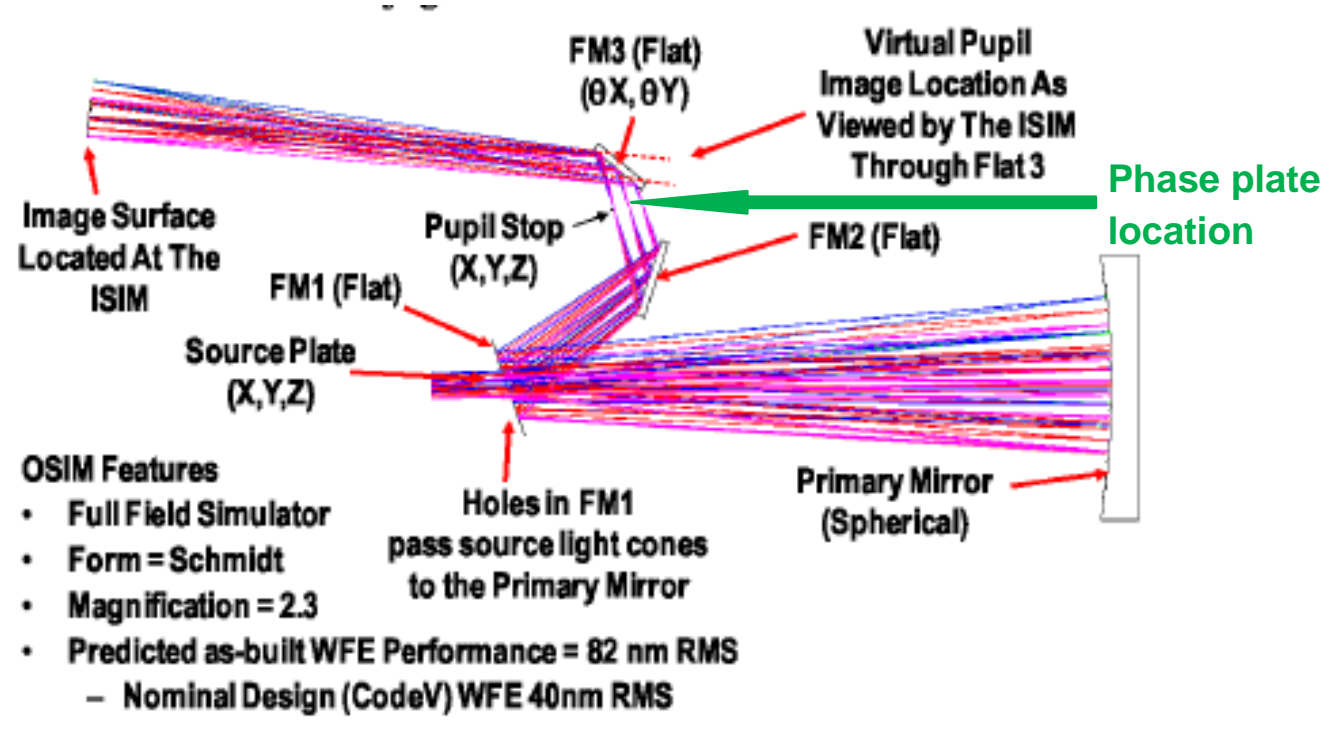

Figure 2. Phase plate location in OSIM.

The location of phase plate in OSIM is shown in Figure 2. It is right on the OSIM pupil stop.

The phase plates were installed in the "pupil select mechanism" shown in Figure 3. This mechanism is configured in such a way that various optical elements can be inserted to OSIM pupil plane through a rotation wheel, including three phase plates. During the DHS algorithm test, the OSIM light source for NIRCam will create an F/20 beam at the phase plate plane. After the beam passes the phase plate, its wavefront is modified by the hexagonal phase pattern to simulate the segmented primary mirror piston error during DHS alignment.
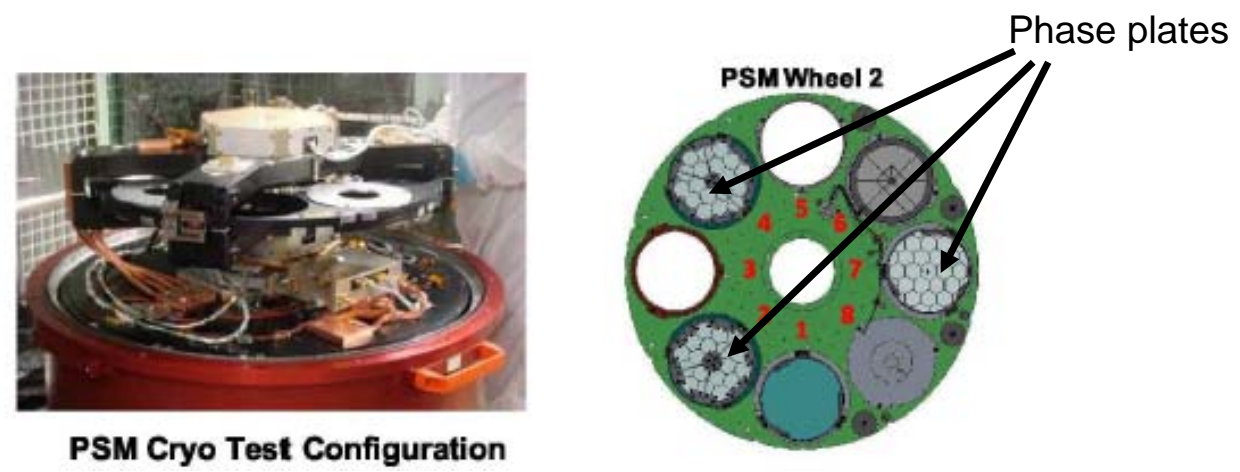

Figure 3. Pupil select mechanism and phase plates.

\subsection{DFS Algorithm}

The DFS algorithm has been studied thoroughly. Especially since it was selected as JWST coarse alignment method to perform on-orbit segmented primary mirror alignment, many papers have been published ${ }^{234}$.

The characterization method for coarse phase place coarse sensing uses the same concept. The main difference between transmitted phase plate discussed here and segmented mirror in other papers is that the dispersion introduced by phase plate material makes the DFS fringes not equally spaced in wave number regime. The detail of how to remove the dispersion effect is addressed in the data analysis section. 


\subsection{DFS test setup}

Optical setup for phase plate DFS measurement is shown in Figure 4. The 5-inch collimated beam was generated by positioning the source (tip of a single mode fiber) at the focal plane of the collimating lens. The coarse phase plate, assembled in a phase plate mechanical wireframe, was positioned in the collimated beam. A DFS mask shown in Figure 4 was inserted right after the phase plate. A grating with a period of 120 cycles / mm was followed after the DFS aperture. The +1 order diffracted light was focused to a CCD by a focusing lens. Due to limited CCD size, the camera was aligned to capture the interference fringes for only the selected spectrum range.
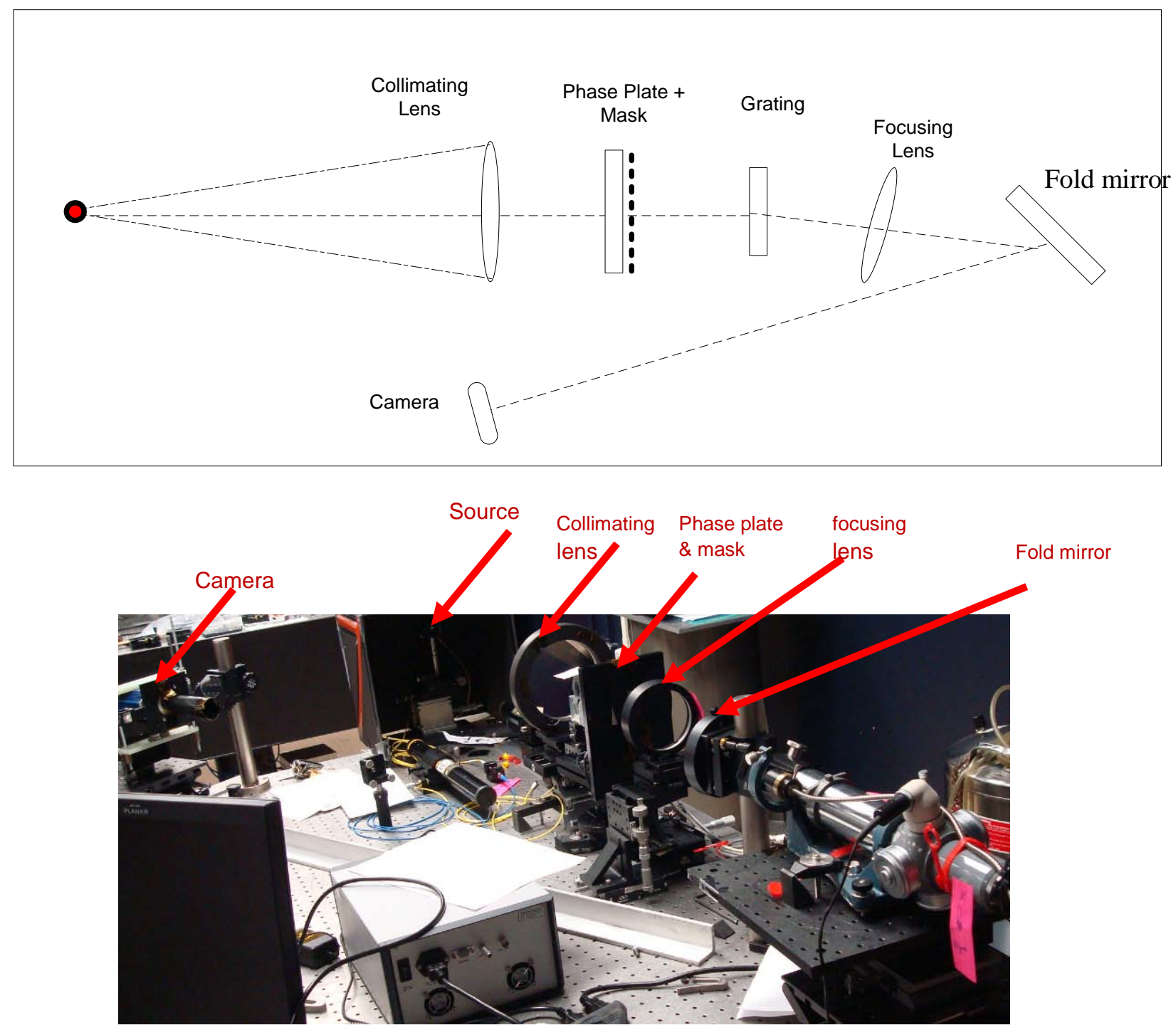

Figure 4. DFS schematic and test setup.

To make the measurement accurate, it is necessary to calibrate the pixel position versus. Fortunately, grating dispersion is linear to wavelength, only two HeNe wavelengths at $594.1 \mathrm{~nm}$ and $632.8 \mathrm{~nm}$ were used for wavelength calibration. After the calibration, the HeNe source was replaced by a super-continuum source through fiber connections to make sure the tip of the fiber was not moved. The super-continuum source continuously covers the wavelength range from above $400 \mathrm{~nm}$ to almost $2000 \mathrm{~nm}$. We only use a small portion in visible. 
The DHS mask has 10 opening apertures. The pattern of the mask is the same as the pattern in NIRCam, but roughly 5 times larger to make sure if the mask is imaged to NIRCam DHS plane, it would overlap NIRCam's DHS mask. Following the NIRCam definition, the vertical orientation (0-degree) was shared by both NIRCamA and NIRCamB. The +60 degrees for NIRCamB and the -60 degrees for NIRCamA as shown in Figure 5.

In Figure 5a, the triacontagon orientation is the same as that of the mask. In Figure $5 b$ and $5 c$, the triacontagon orientation has a 60 degree angle with that of the mask.

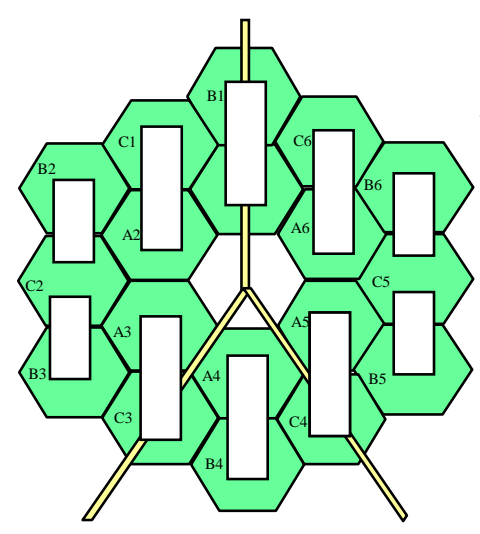

(a)

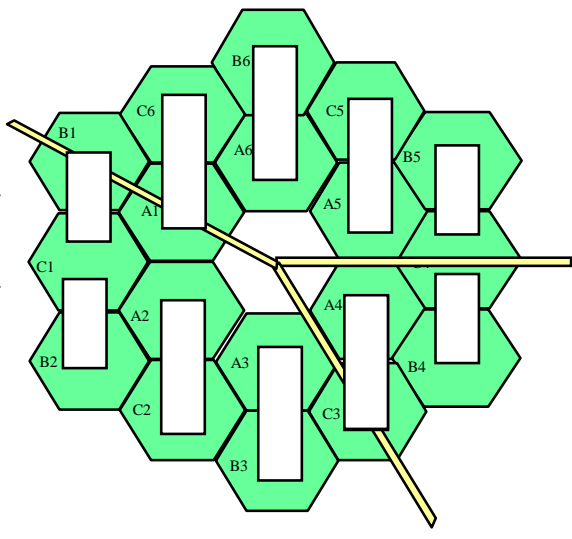

(b)

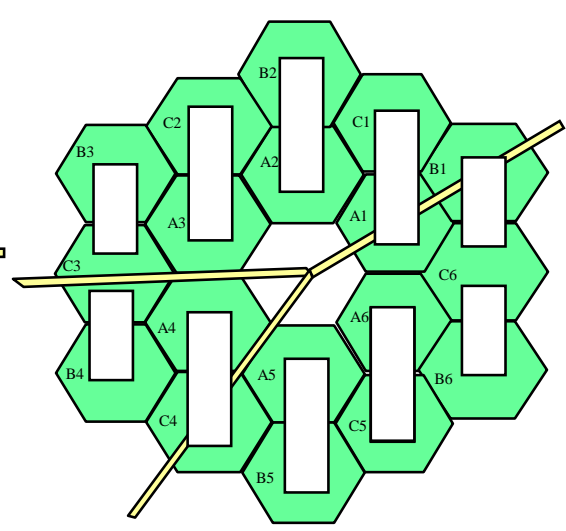

(c)

Figure 5. DHS mask for the coarse phase plate at three orientations to simulate NIRCamA\&B performance.

\section{FUSED SILICA PHASE PLATE CHARACTERIZATION}

\subsection{DFS Fringes}

The typical measured DFS data is shown in Figure 6.

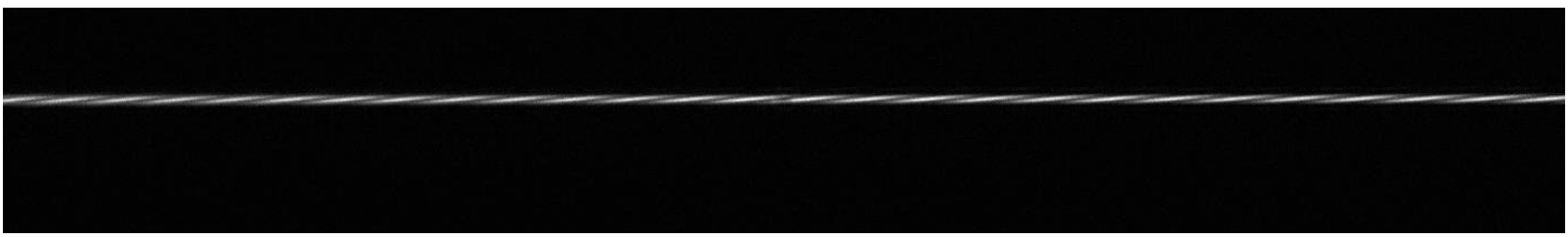

(a) Complete fringes on CCD

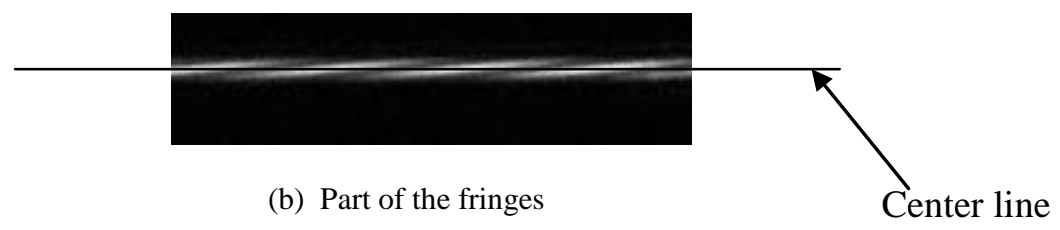

Figure 6. Measured DHS data

In figure 6 (b), the three rows around the horizontal center line were selected to perform piston analysis. Figure 7 is an intensity distribution plot from the center line. 


\section{A sample of raw data}

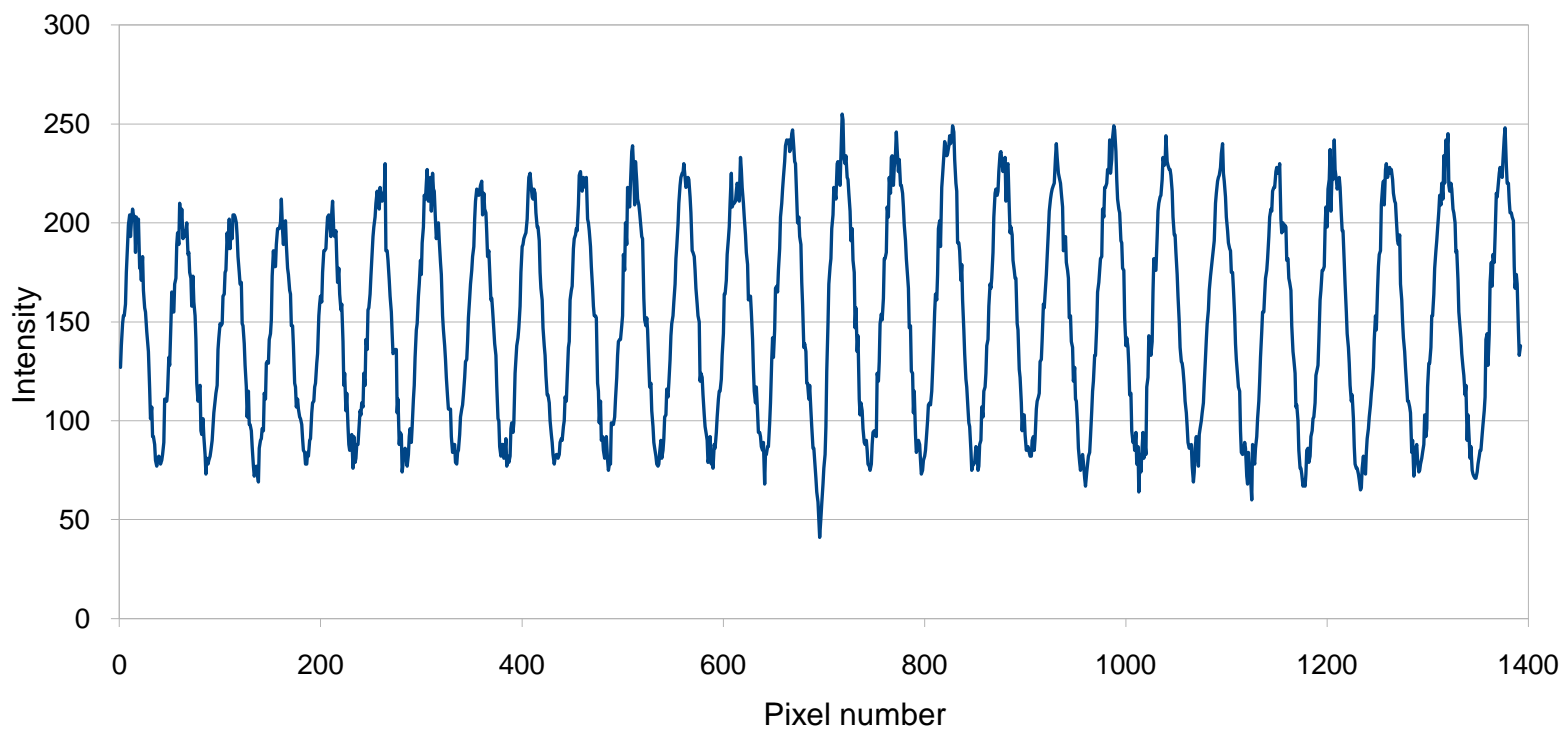

Figure 7. DHS central line intensity distribution.

\subsection{Dispersion Characteristics of Fused Silica Phase plate}

Two different algorithms were used for this analysis. Because the coarse phase plate is made of Corning 7980 fused silica, its spectral dispersion makes the piston a function of wavelength. So the first step of the analysis is to remove the dispersion effect.

As mentioned above, the phase plate is made of fused silica. Assume the thickness difference between the two adjacent segments is $\Delta \mathrm{t}$, the piston $\Delta \mathrm{p}$ will be:

$$
\Delta p(\lambda)=\Delta t(n(\lambda)-1)
$$

For Corning 7980 fused silica,

$$
n(\lambda)=A_{0}+A_{1} \lambda^{4}+A_{2} \lambda^{2}+A_{3} \lambda^{-2}+A_{4} \lambda^{-4}+A_{5} \lambda^{-6}+A_{6} \lambda^{-8}+A_{7} \lambda^{-10}
$$

where

$$
\begin{aligned}
& A_{0}=2.104025406 \\
& A_{1}=-1.456000330 \times 10^{-4} \\
& A_{2}=-9.049135390 \times 10^{-3} \\
& A_{3}=8.801830992 \times 10^{-3} \\
& A_{4}=8.435237228 \times 10^{-5} \\
& A_{5}=1.681656789 \times 10^{-6} \\
& A_{6}=-1.675425449 \times 10^{-8} \\
& A_{7}=8.326602461 \times 10^{-10}
\end{aligned}
$$


As we know, the piston between any two JWST mirror segments is wavefront independent. In order to use phase plate to verify JWST algorithm, the dispersion effect of the phase plate needs to be removed. This is done by adjusting the scale of the DFS data as follows:

If the dispersion did not exist, the wavelengths at the peaks would be:

$$
\lambda_{\text {Peak }}=\frac{\Delta p}{2 m \pi}=\frac{\Delta t\left(n_{c}-1\right)}{2 m \pi}, \quad m=1,2,3, \ldots
$$

Assume $\mathrm{n}_{\mathrm{c}}$ is the refractive index of the central wavelength $\lambda_{\mathrm{c}}$, and $\lambda_{\text {Peak }}$ is only a function of $\mathrm{m}$.

When the dispersion exists, the peak wavelengths become:

$$
\lambda_{\text {Peak }}=\frac{\Delta p}{2 m \pi}=\frac{\Delta t(n(\lambda)-1)}{2 m \pi}, \quad m=1,2,3,
$$

Now, $\lambda_{\text {Peak }}$ is not only the function of $\mathrm{m}$, it is also the function of wavelength. It is important to mention that for the transmitted phase plate, talking about piston itself is not very meaningful. The correct way is to say piston at a given wavelength. All result from this paper is referenced to $\lambda_{\mathrm{c}}=1.0 \mu \mathrm{m}$.

Comparing the two equations above, it is obvious that the data taken from phase plate has to be stretched by a function of $\left(n_{c}-1\right) /(n(\lambda)-1)$. So before calculating piston, the original dispersion axis that is linear to wavelength is first multiplied by a function of $\left(n_{c}-1\right) /(n(\lambda)-1)$. Then the data is interpolated to the value at original wavelengths. For example, the raw data in pixel $i$ is the intensity of $\lambda_{\mathrm{i}}$, after correction, it becomes the intensity of $\lambda_{\text {new }, \mathrm{i}}=\lambda_{\mathrm{i}} \times\left(n_{c}-1\right) /(n(\lambda)-1)$. However, the data points to be processed need to be linear to wavelength, so the intensity will be interpolated to original wavelength points.

Figure 8 shows two simulated DFS curves with the same piston value $199.402 \mu \mathrm{m}$ at the central wavelength: one is for mirror case, and the other is for fused silica case. The difference between the two curves is obvious.

\section{Comparison of mirror generated and fused silica generated DFS curves}

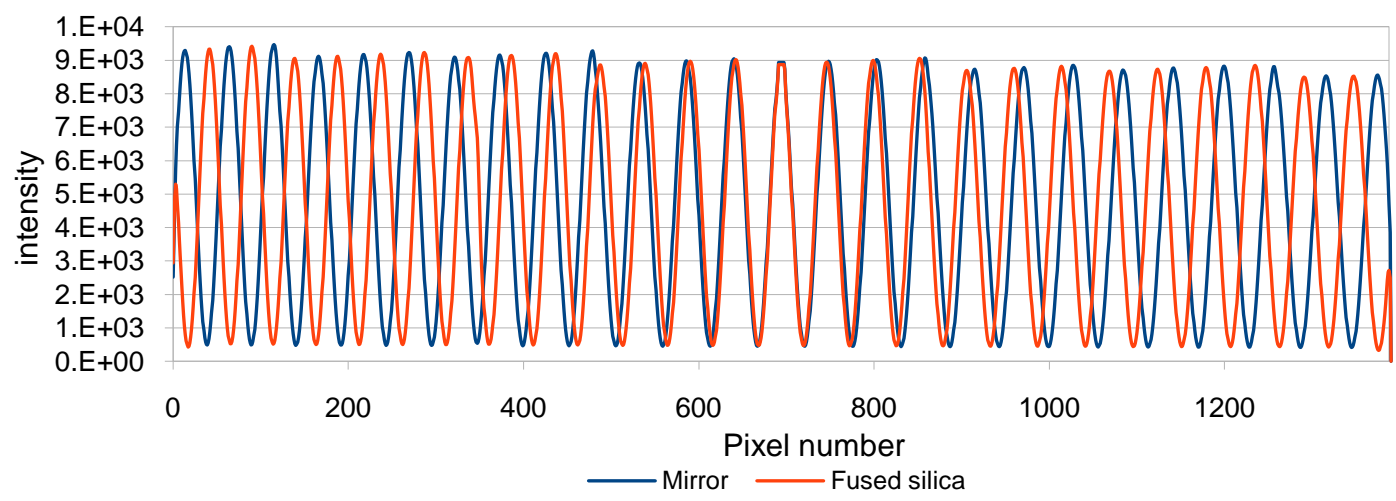

Figure 8. Comparison of DFS curve with and without dispersion.

Figure 9 shows the measured DFS curve and simulated DFS curve. The piston from the measured curve is $199.402 \mu \mathrm{m}$. A simulated curve is also plotted in the same figure. It is seen that the two curves are matched very well in the dispersion direction. 


\section{Comparison of measured and simulated}

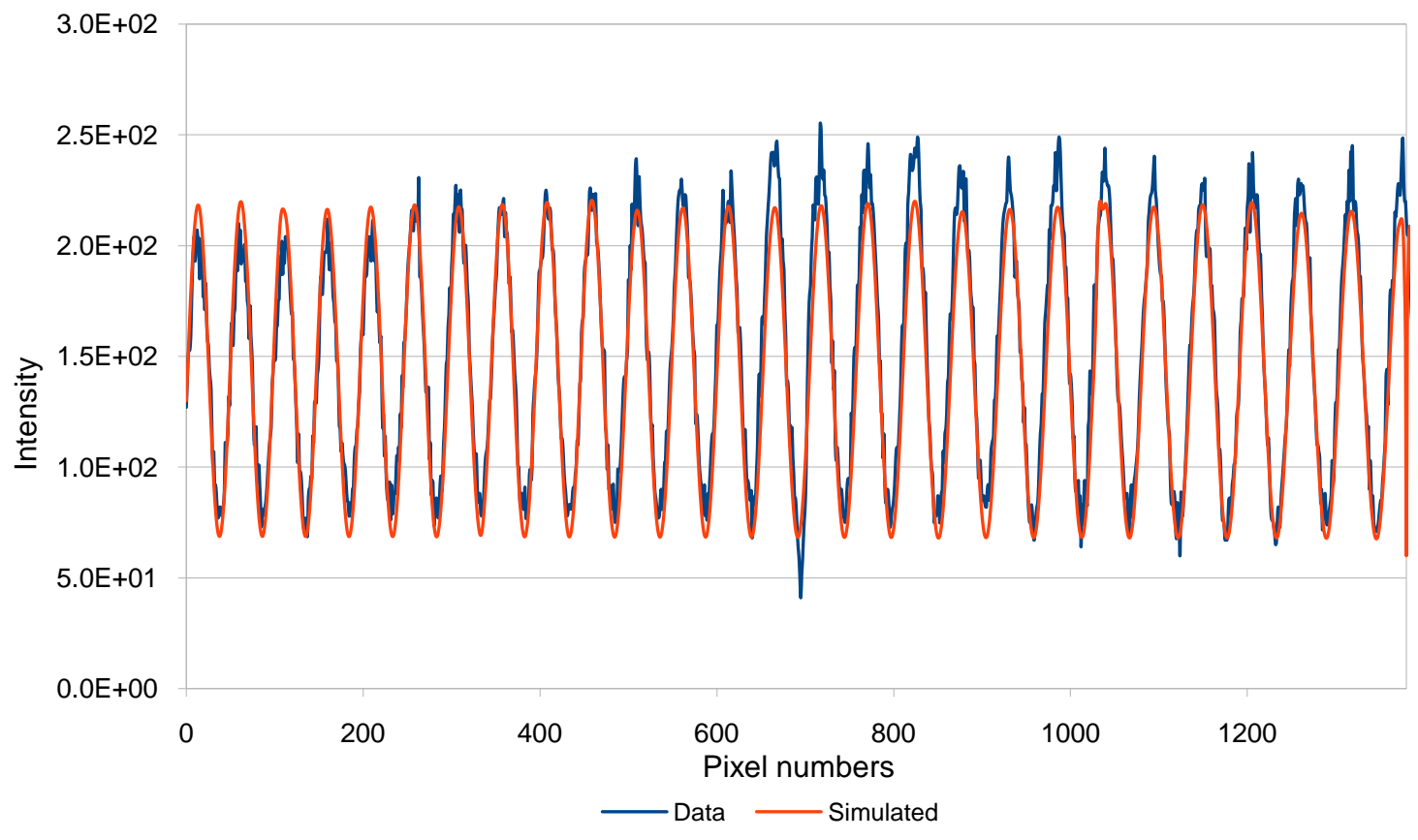

Figure 9. Typical measured and simulated DFS curves.

During the piston retrieval, the raw data was first post-processed to remove the noise and to make the amplitude flat over the whole curve. Then it was corrected for the dispersion introduced by fused silica. Figure 10 shows the measured DFS curve after post-process and the fitted curve. It is noted that the two curves are very close.

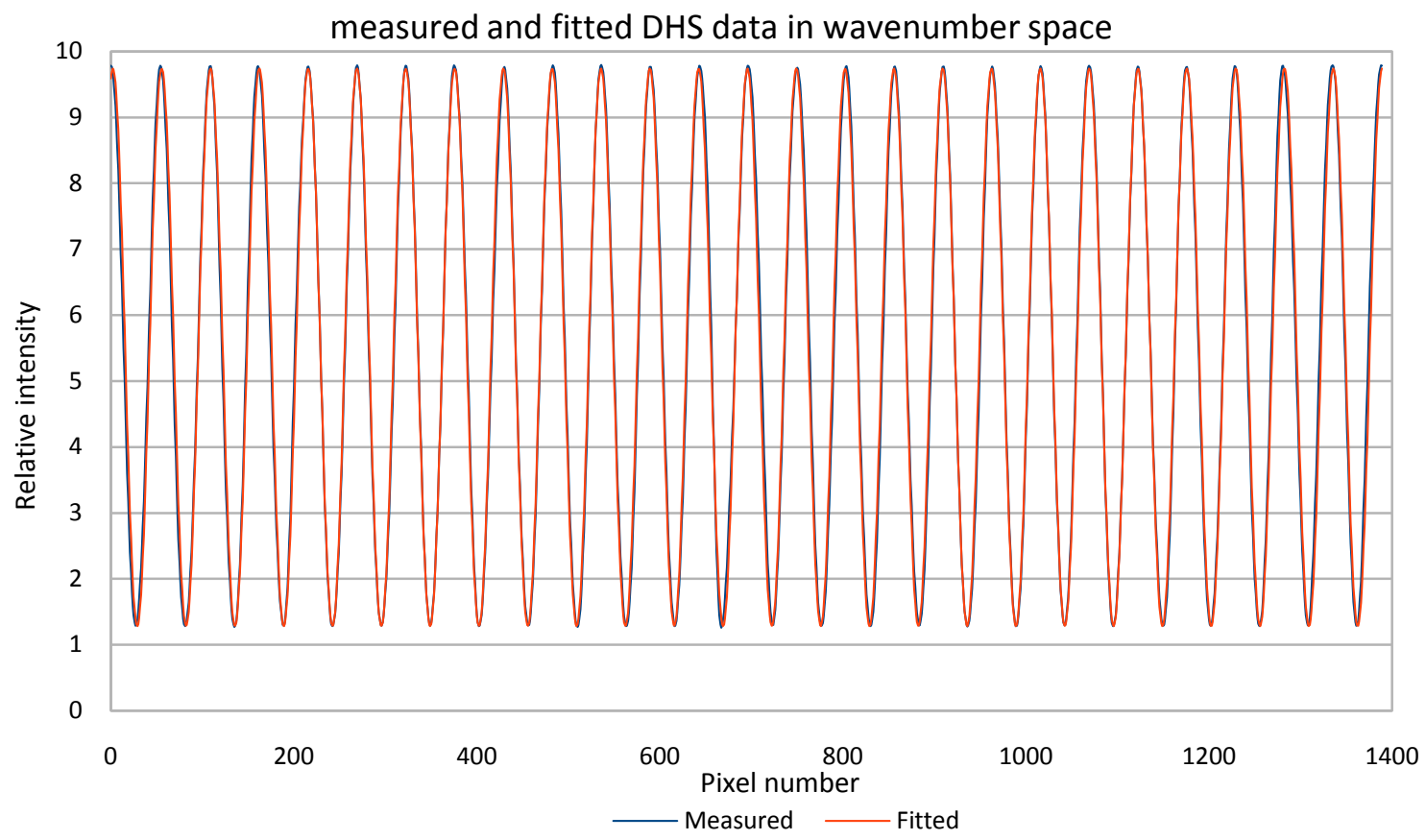

Figure 10. Post-processed measurement curve and fitted curve. 


\section{DATA ANALYSIS AND RESULT}

The designed coarse phase plate piston distribution was previously shown in Figure 1. Due to fabrication tolerance, the differences exist between real and designed values. The tolerance itself is not critical, as long as the true piston differences between any two adjacent segments are well characterized.

The piston characterization here uses two different methods: FFT based modeling and Sine Wave fit. Both methods convert the data from wavelength regime to wavenumber regime to make the dispersed fringes equally spaced. The FFT method uses the frequency after FFT to determine the piston difference between the pair of segments. The Sine wave fit method uses least squares fit to find the frequency of sine function that has the best fit to DFS data. Between the two methods, the sine wave fit method is believed to be more accurate when frequency (the piston value) is relatively low. Figure 11 (a) shows the sine wave fit with both original data and fitted curve. In this plot, 1 cycle is about $4.1 \mu \mathrm{m}$ of mirror piston. The estimation from the plot shows that the uncertainty would not exceed $5 \%$, that is, the piston error is not going to be more than $0.2 \mu \mathrm{m}$. The FFT from the same data set is shown in Figure 11. Because the frequency is too close to 0 , it is difficult to locate the peak accurately.

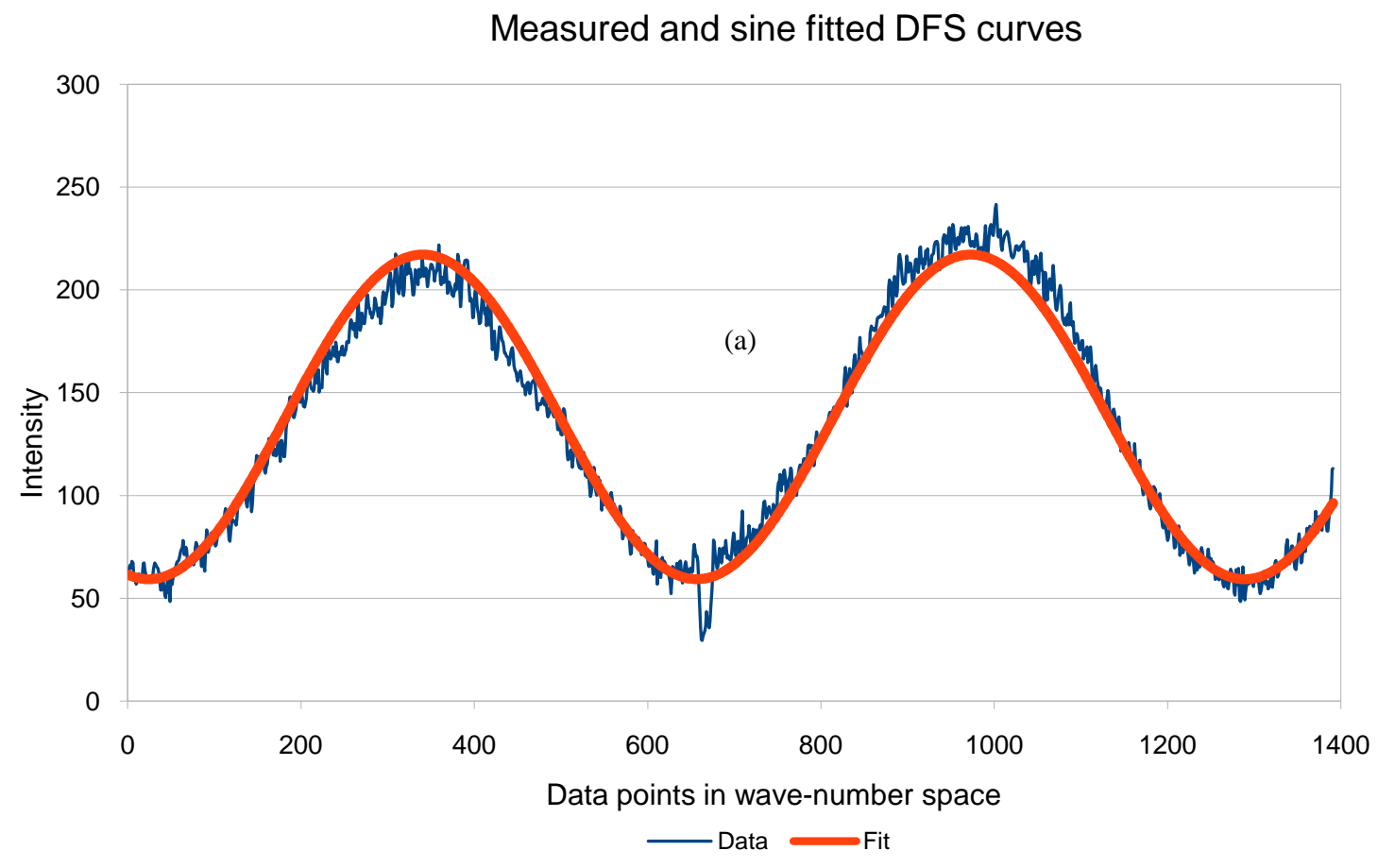

Figure 11. (a) Sine wave fit 


\section{FFT of DFS data}

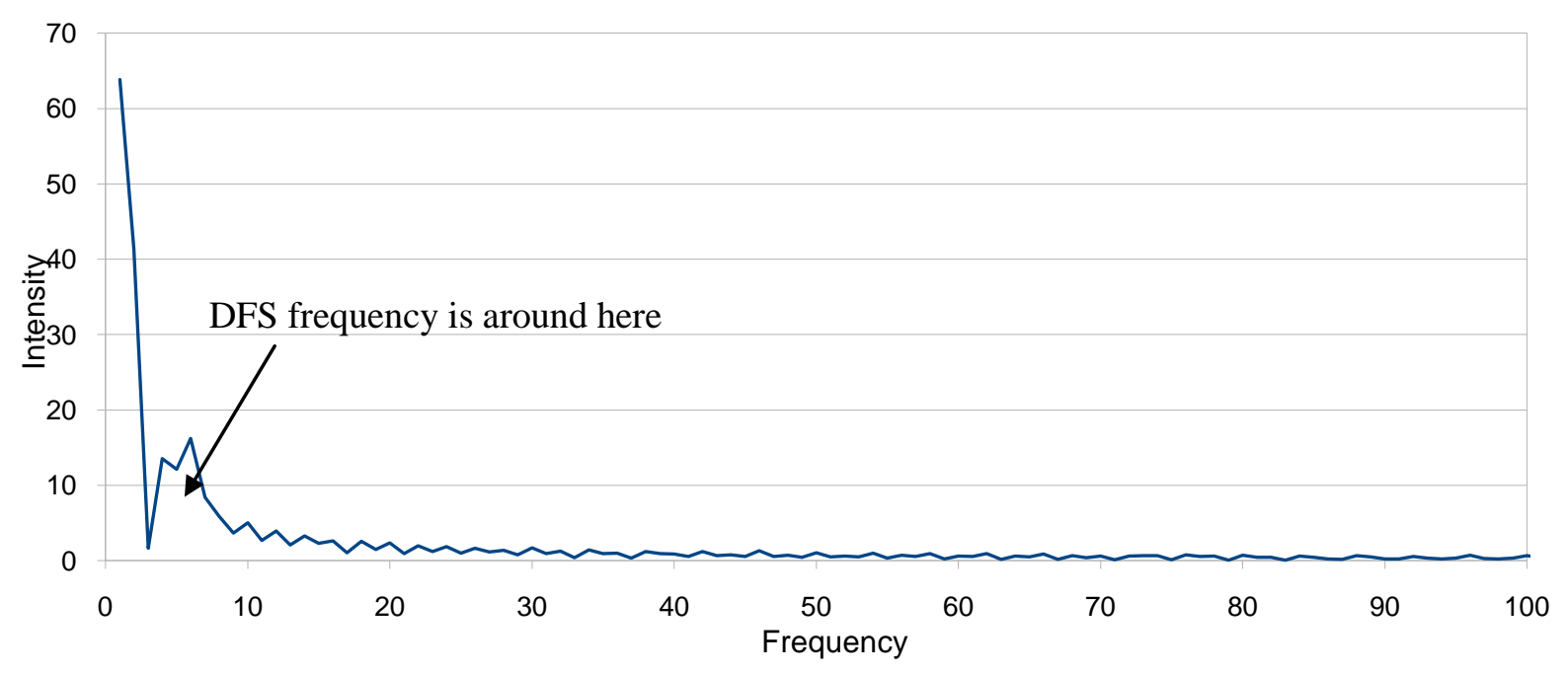

(b) DFS after FFT

Figure 11. FFT and sine wave fit comparison

The DFS measurement of coarse phase plate piston includes two different configurations: NIRCamA and NIRCamB, which reflects the DHS measurement of NIRCam instrument described in test setup section.

The globalization of piston starts from segment B5. The piston of B5 is set to zero. The first set of piston data uses vertical pairs and $+60^{\circ}$ pairs for NIRCamA (see test setup section). The globalization follows the path indicated by arrows in Figure 12. The measured piston for each segment is listed in Table 1. The second set of piston data uses vertical pairs and $-60^{\circ}$ pairs for NIRCamB. The globalization follows the path indicated by arrows in Figure 13 . The measured piston for each segment is listed in Table 2 . 


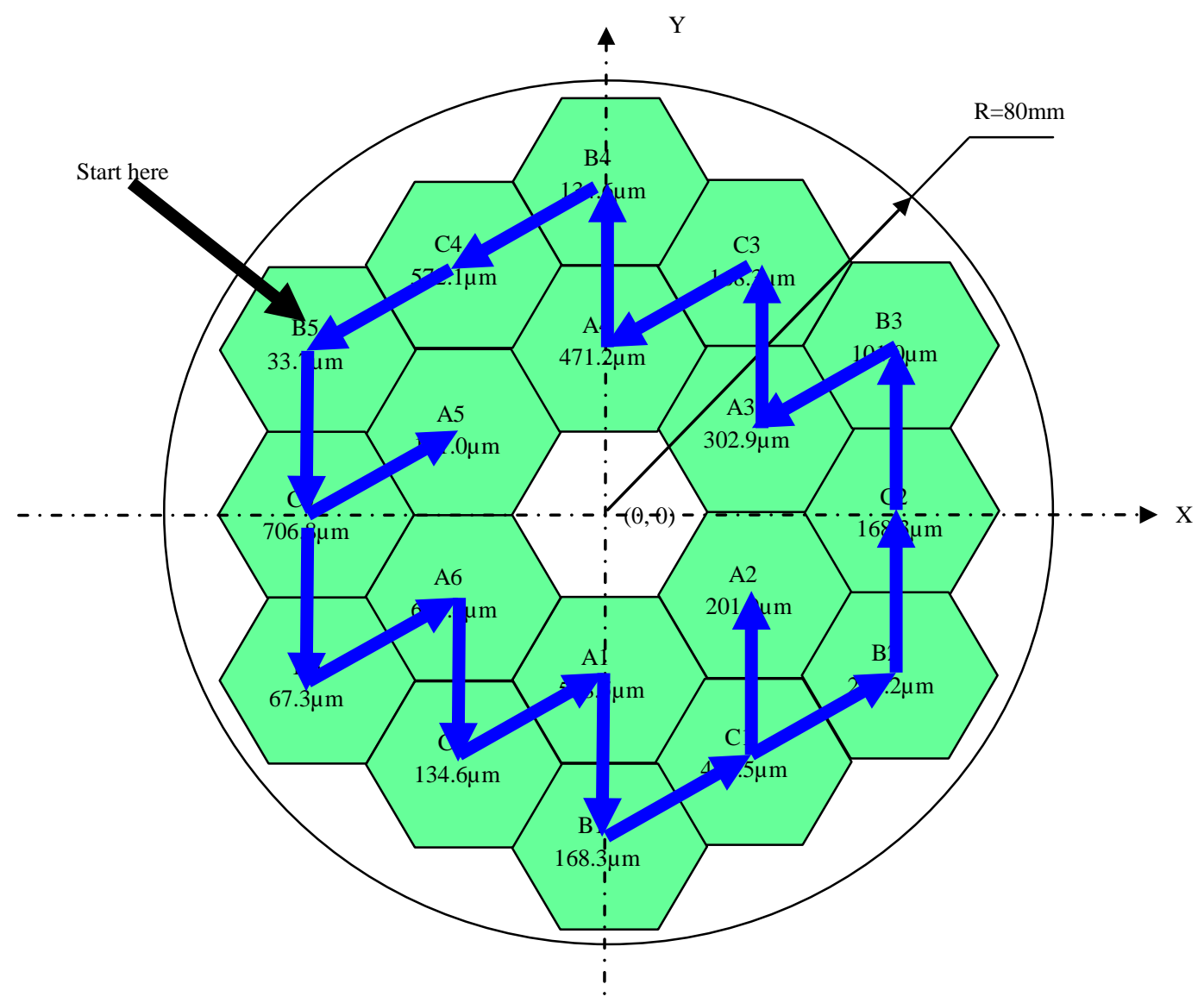

Figure 12. Vertical and $60^{\circ}$ pair for NIRCamA global piston calculation path.

Table 1. Piston values calculated using FFT and sine wave fit for NIRCamA position. (Wavefront piston $=2 \times$ mirror piston)

\begin{tabular}{|l|r|r|r|r|}
\hline & \multicolumn{3}{|l|}{ Wavefron piston $(\mu \mathrm{m})$} & \multicolumn{3}{l|}{ Mirror piston $(\mu \mathrm{m})$} \\
\hline Segment & \multicolumn{1}{|l|}{ Sine Fit } & \multicolumn{1}{l|}{ FFT } & \multicolumn{1}{l|}{ FFT } \\
\hline B5 & 0 & 0 & 0 & 0 \\
\hline C5 & 312.41 & 312.49 & 156.20 & 156.24 \\
\hline B6 & 28.47 & 28.79 & 14.23 & 14.40 \\
\hline A5 & 37.84 & 37.80 & 18.92 & 18.90 \\
\hline A6 & 294.96 & 295.30 & 147.48 & 147.65 \\
\hline C6 & 17.17 & 17.52 & 8.58 & 8.76 \\
\hline A1 & 254.48 & 254.83 & 127.24 & 127.41 \\
\hline B1 & 59.08 & 59.42 & 29.54 & 29.71 \\
\hline C1 & 199.64 & 200.17 & 99.82 & 100.09 \\
\hline A2 & 94.29 & 94.86 & 47.15 & 47.43 \\
\hline B2 & 119.74 & 120.38 & 59.87 & 60.19 \\
\hline C2 & 56.95 & 57.85 & 28.47 & 28.92 \\
\hline B3 & 38.96 & 41.77 & 19.48 & 20.89 \\
\hline A3 & 136.57 & 139.21 & 68.28 & 69.61 \\
\hline C3 & 55.97 & 58.61 & 27.98 & 29.31 \\
\hline A4 & 213.30 & 215.95 & 106.65 & 107.98 \\
\hline B4 & 40.93 & 43.56 & 20.47 & 21.78 \\
\hline C4 & 251.28 & 254.02 & 125.64 & 127.01 \\
\hline B5 & -0.74 & 1.92 & -0.37 & 0.96 \\
\hline
\end{tabular}




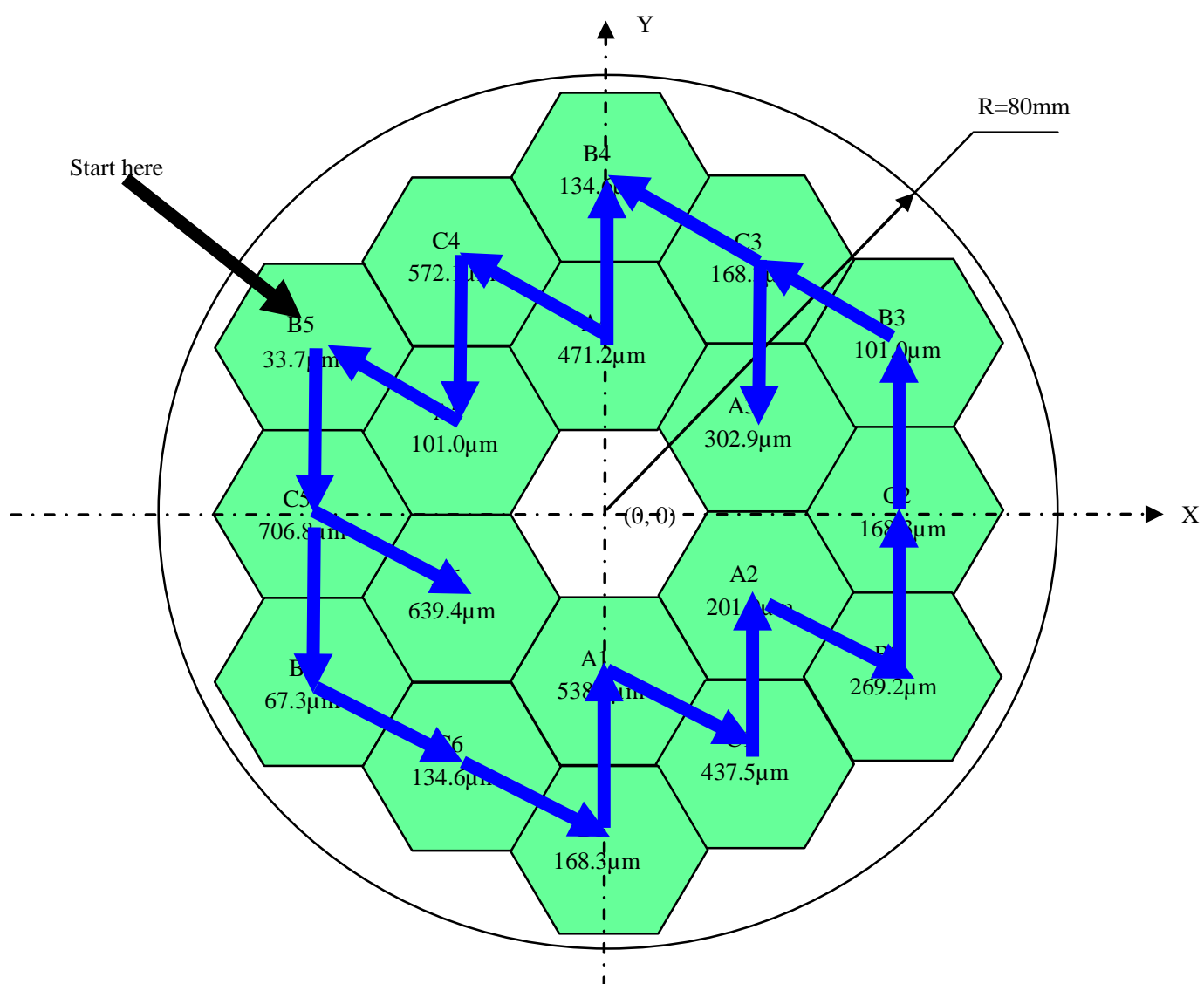

Figure 13. Vertical and $-60^{\circ}$ pair'for NIRCamB global piston calculation path.

Table 2. Piston values calculated using FFT and sine wave fit for NIRCamB position. (Wavefront piston $=2 \times$ mirror piston)

\begin{tabular}{|l|r|r|r|r|}
\hline & \multicolumn{3}{|l|}{ lavefron piston $(\mu \mathrm{m})$} & \multicolumn{3}{l|}{ Mirror piston $(\mu \mathrm{m})$} \\
\hline Segment & \multicolumn{1}{|l|}{ Sine Fit } & \multicolumn{1}{l|}{ FFT } & \multicolumn{1}{l|}{ Sine Fit } & \multicolumn{1}{l|}{ FFT } \\
\hline B5 & 0.00 & 0.00 & 0.00 & 0.00 \\
\hline C5 & 312.41 & 312.49 & 156.20 & 156.24 \\
\hline A6 & 289.19 & 288.91 & 144.59 & 144.45 \\
\hline B6 & 28.47 & 28.79 & 14.23 & 14.40 \\
\hline C6 & 11.39 & 11.12 & 5.70 & 5.56 \\
\hline B1 & 54.70 & 54.44 & 27.35 & 27.22 \\
\hline A1 & 250.11 & 249.84 & 125.05 & 124.92 \\
\hline C1 & 196.60 & 195.74 & 98.30 & 97.87 \\
\hline A2 & 91.25 & 90.42 & 45.62 & 45.21 \\
\hline B2 & 116.36 & 115.87 & 58.18 & 57.93 \\
\hline C2 & 53.56 & 53.33 & 26.78 & 26.67 \\
\hline B3 & 35.57 & 37.25 & 17.79 & 18.63 \\
\hline C3 & 54.60 & 54.80 & 27.30 & 27.40 \\
\hline A3 & 135.21 & 135.40 & 67.60 & 67.70 \\
\hline B4 & 38.59 & 38.70 & 19.30 & 19.35 \\
\hline A4 & 210.97 & 211.10 & 105.48 & 105.55 \\
\hline C4 & 250.50 & 250.04 & 125.25 & 125.02 \\
\hline A5 & 36.88 & 36.35 & 18.44 & 18.18 \\
\hline B5 & -1.59 & -1.64 & -0.80 & -0.82 \\
\hline
\end{tabular}


The full loop starts from segment B5, and ends at the same segment B5. From Tables 1 and 2, it is noted that the uncertainty in wavefront piston is less than $2 \mu \mathrm{m}$, and in mirror piston is less than $1 \mu \mathrm{m}$. This error is well within the required $4 \mu \mathrm{m}$ to start fine phasing using PDI. This error is due to measurement setup, data quality, and analysis process.

Table 3 shows that piston difference between NIRCam A and B globalizations. It is noted that this difference is much larger than either NIRCamA or NIRCamB. This difference is believed largely due to segment tilts. After a full circle, the tilt should be canceled. But the accumulated tilt that contributes to piston at any particular segment stays there. The following PDI analysis will provide more decisive conclusion on this.

Table 3. Piston differences from NIRCamA \& B after globalization.

\begin{tabular}{|l|r|r|r|r|r|r|}
\hline & \multicolumn{3}{|c|}{ Wavefront piston $(\mu \mathrm{m})$} & \multicolumn{3}{c|}{ Mirror piston $(\mu \mathrm{m})$} \\
\cline { 2 - 7 } & NIRCamA & NIRCamB & Abs $(\Delta(\mathrm{A}-\mathrm{B}))$ & NIRCamA & NIRCamB & \multicolumn{1}{c|}{ Abs $(\Delta(\mathrm{A}-\mathrm{B}))$} \\
\hline A1 & 254.48 & 250.11 & 4.38 & 127.24 & 125.05 & 2.19 \\
$\mathrm{~A} 2$ & 94.29 & 91.25 & 3.04 & 47.15 & 45.62 & 1.52 \\
A3 & 136.57 & 135.21 & 1.36 & 68.28 & 67.60 & 0.68 \\
A4 & 213.30 & 210.97 & 2.34 & 106.65 & 105.48 & 1.17 \\
A5 & 37.84 & 36.88 & 0.97 & 18.92 & 18.44 & 0.48 \\
A6 & 294.96 & 289.19 & 5.78 & 147.48 & 144.59 & 2.89 \\
B1 & 59.08 & 54.70 & 4.38 & 29.54 & 27.35 & 2.19 \\
B2 & 119.74 & 116.36 & 3.38 & 59.87 & 58.18 & 1.69 \\
B3 & 38.96 & 35.57 & 3.38 & 19.48 & 17.79 & 1.69 \\
B4 & 40.93 & 38.59 & 2.34 & 20.47 & 19.30 & 1.17 \\
B5 & 0.00 & 0.00 & 0.00 & 0.00 & 0.00 & 0.00 \\
B6 & 28.47 & 28.47 & 0.00 & 14.23 & 14.23 & 0.00 \\
C1 & 199.64 & 196.60 & 3.04 & 99.82 & 98.30 & 1.52 \\
C2 & 56.95 & 53.56 & 3.38 & 28.47 & 26.78 & 1.69 \\
C3 & 55.97 & 54.60 & 1.36 & 27.98 & 27.30 & 0.68 \\
C4 & 251.28 & 250.50 & 0.78 & 125.64 & 125.25 & 0.39 \\
C5 & 312.41 & 312.41 & 0.00 & 156.20 & 156.20 & 0.00 \\
C6 & 17.17 & 11.39 & 5.78 & 8.58 & 5.70 & 2.89 \\
\hline
\end{tabular}

\section{SUMMARY}

The phase plates are critical optical elements to verify JWST's on-orbit segmented mirror mechanism. How well the phase plates can be characterized directly relates to how well the mechanism can be verified. The DFS test result shows that the initial coarse phasing of the coarse phase plate has exceeded the original accuracy requirement (4 $\mu \mathrm{m}$ uncertainty for DFS coarse phasing). It has established a solid ground for the next step - using the PDI for fine phasing. The PDI test will collect interferograms at 3 selected wavelengths to further reduce the uncertainty to nanometer range, and to characterize the wavefront errors of each segment and the whole phase plate. As mentioned in the introduction section, it will be done in the near future and the final result will be reported then.

\section{ACKNOWLEDGEMENTS}

We would like to thank all OSIM team at GSFC and Ball Aerospace for their support to make this coarse phase plate characterization possible. Special thanks to the mechanical team for assembling 19 segments with very demanding tolerances. Special thanks also go to ProSystem, Inc. for fabricating the segment with high quality wavefront and tight thicknesses requirement. 


\section{REFERENCES}

${ }^{1}$ J. Sullivan, et al. "Manufacturing and Integration Status of the JWST OSIM Optical Simulator”, Proc. SPIE 7731 (2010).

${ }^{2}$ L. Feinberg et al. "TRL-6 for JWST wavefront sensing and control". Proceedings of SPIE vol. 6687 (2008)

${ }^{3}$ F. Shi, et al. "Experimental verification of dispersed fringe sensing as a segment phasing technique using the Keck telescope”, APPLIED OPTICS _ Vol. 43, No. 23 _ 10 August 2004

${ }^{4}$ F. Shi, et al. "Performance of dispersed fringe sensor in the presence of segmented mirror aberrations - modeling and simulation”, Pasadena, CA : Jet Propulsion Laboratory, National Aeronautics and Space Administration, 2006. 